\title{
Staged hybrid repair of a Kommerell diverticulum in conjunction with double thoracic saccular aortic aneurysms
}

\author{
Tomoki Nagata ${ }^{1}$, Takashi Yamada ${ }^{2}$, Shinichi Iwakoshi ${ }^{3}$, and Hiroyuki Johno ${ }^{2}$ \\ ${ }^{1}$ Takaishi Fujii Cardiovascular Hospita \\ ${ }^{2}$ Takaishi Fujii Cardiovascular Hospital \\ ${ }^{3}$ Nara Medical University
}

April 28, 2020

\begin{abstract}
Although a Kommerell diverticulum (KD) and aberrant subclavian artery (ASA) are a common congenital anomaly, a KD and ASA with two thoracic aortic aneurysms are rarely reported. We describe a case of a KD with a right ASA and two thoracic saccular aortic aneurysms, which were treated with a total arch replacement, frozen elephant trunk technique, and thoracic endovascular aortic repair.
\end{abstract}

\section{Introduction}

A Kommerell diverticulum (KD) is a complex congenital anomaly of the aorta. It is asymptomatic but has a risk of a rupture or dissection. Several options have been described to treat an aberrant subclavian artery (ASA) and associated KD. We report a rare case of a successful staged hybrid open repair of a KD and two thoracic saccular aortic aneurysms.

\section{Case presentation}

An 83-year-old man presented with a KD and right ASA originating from the descending aorta as the fourth supra-aortic branch passing behind the trachea and esophagus. The KD measured $21 \mathrm{~mm}$. Additionally, he had an 83-mm thoracic saccular aneurysm on the aortic arch and a 45-mm saccular aneurysm on the descending aorta (Figure 1).

We planned a two staged hybrid procedure using a total arch replacement (TAR) with a frozen elephant trunk (FET) and distal extension of the thoracic endovascular aortic repair (TEVAR).

The patient underwent a TAR using the FET technique performed through a median full sternotomy. Initially, $9 \mathrm{~mm}$ tubular grafts (J Graft Japan Lifeline Co, Ltd, Tokyo, Japan) were anastomosed to both the left and right axillar arteries in their second portion. After systemic heparinization, an arterial cannula was inserted from the ascending aorta, and venous cannulas were placed through the superior and inferior vena cavae. Subsequently, cardiopulmonary bypass (CPB) was instituted. The right ASA was exposed just at the right side of the main bronchus with the guidance of preoperative $\mathrm{CT}$.

As the core temperature fell to $30{ }^{\circ} \mathrm{C}$, under circulatory arrest, the right ASA was ligated at the previous dissected part. After the left subclavian artery was ligated at its origin, antegrade selective cerebral perfusion (SCP) was initiated through both of the $9 \mathrm{~mm}$ graft conduits anastomosed to both axillar arteries. TwelveFr balloon-tipped cannulas were inserted into both common carotid arteries. The antegrade SCP flow was maintained at 10 to $12 \mathrm{ml} / \mathrm{kg} / \mathrm{min}$ using an independent roller pump, and the balloon tip pressure was maintained between 30 and $40 \mathrm{mmHg}$. 
The aortic arch was transected between the left common carotid and left subclavian artery, and a $31-120$ mm FET (J graft FROZENIX Japan Lifeline Co, Ltd, Tokyo, Japan) was inserted with direct vision without fluoroscopy or trans-esophageal echocardiography. After deploying the FET, a stump of the graft was anastomosed to the four-branched graft (J Graft Japan Lifeline Co, Ltd, Tokyo, Japan) by a 3-0 polypropylene running suture. The lower body circulation was reinstituted through a branch graft. The proximal anastomosis with a 3-0 polypropylene running suture was then accomplished, followed by coronary reperfusion. The first branch was anastomosed to the right carotid artery and the second to the left carotid artery by a 4-0 polypropylene running suture. A 9-mm tubular graft from the right subclavian artery was anastomosed to the first side branch using an end-to-side anastomosis. Finally, another 9-mm tubular graft from the left subclavian artery was anastomosed to the ascending tubular graft in an end-to-side fashion. The durations of the operation, extracorporeal circulation, aortic cross-clamping, and circulatory arrest were 391, 185, 95, and 74 minutes, respectively. Postoperative CT revealed good patency of the bypass graft to the supra-aortic vessels and complete exclusion of any KD or saccular aneurysm by the FET (Figure 2).

Three weeks after the TAR with an FET, a scheduled TEVAR was performed. A stent graft (Gore CTAG $26 \mathrm{~mm} \times 200 \mathrm{~mm}$ Gore \& Associates, Flagstaff, AZ, USA) was deployed in the lower descending aorta in a retrograde fashion through a left femoral access. Subsequently, the second stent graft (Gore CTAG $31 \mathrm{~mm}$ $\times 200 \mathrm{~mm}$ Gore \& Associates, Flagstaff, AZ, USA) was additionally inserted as a bridging of the previously placed FET and first stent graft.

The patient's recovery was uneventful. The postoperative CT showed the complete exclusion of both aneurysms and KD by the stent graft (Figure 3). He was discharged from the hospital 10 days after the surgery without any complications.

\section{Discussion}

This case report showed a rare condition with a combination of a Kommerell diverticulum (KD) and two thoracic aortic aneurysms, which were treated by combination of open surgical repair and thoracic endovascular aortic repair (TEVAR). An aberrant subclavian artery (ASA) results from a developmental error in the fourth aortic arch, and persistence of a left-sided or right-sided aortic arch results in a right or left ASA, respectively [1]. The prevalence of a right ASA is $0.5 \%$ to $2 \%$ [2]. In general patients with a right ASA are asymptomatic. However, some patients present with dyspnea, dysphagia stridor, wheezing, coughing, choking spells, recurrent pneumonia, obstructive emphysema, and chest pain, because the right ASA passes behind the esophagus (80\%), between the esophagus and the trachea (15\%), or in front of the trachea $(5 \%)$ [3]. A dilation and aneurysm at the origin of the ASA is known as Kommerell diverticulum (KD) and carries a high risk of rupture or dissection. Although there is no established management guideline because of its rarity, surgical intervention is indicated in patients with symptoms or an aneurysm to prevent a rupture [1].

Surgical, endovascular, or hybrid procedures have been described to treat an ASA and KD. An endovascular approach is less invasive than open surgery and has recently become a common procedure. However, in our case, an endovascular repair was not suitable because of the short landing zone and enlargement of the ascending arch (diameter of $42 \mathrm{~mm}$ ). Therefore, we decided to perform a TAR with an FET. This approach is safe and effective and allows avoidance of a left thoracotomy and resection of the distal arch, and it has the advantage of avoiding recurrent nerve injury [4-7]. A second-stage TEVAR for a residual aneurysm at the descending artery was successfully performed 3 weeks after the TAR with an FET without spinal cord ischemia. Although there was a risk of a rupture of a residual aneurysm during the interval between procedures, we believed that our staged approach reduced the incidence of spinal cord ischemia. Some reports on the treatment of a KD and ASA have been reported thus far in the literature. However, it is considered rare to find two thoracic saccular aortic aneurysms coexisting with a $\mathrm{KD}$, as was discovered in this patient.

\section{Conclusion}

A KD and right ASA are a congenital arterial anomaly. A KD in a patient whose aneurysms are located both in the aortic arch and descending thoracic artery is rarely seen. We successfully performed a TAR with an FET and TEVAR for a KD and double thoracic saccular aortic aneurysms. 


\section{Authors contribution}

TN drafted the manuscript. TY and SI edited and revised the manuscript for the important intellectual content. All authors read and approved the final manuscript.

\section{References}

1) E. Kieffer, A. Bahnini, F. Koskas. Aberrant subclavian artery: surgical treatment in thirty-three adult patients. J Vasc Surg 1994;19:100-109.

2) R. Kopp, I. Wizgall, E. Kreuzer, G. Meimarakis, R.Weidenhagen, A. Kühnl, et al. Surgical and endovascular treatment of symptomatic aberrant right subclavian artery (arteria lusoria)

Vascular 2007;15:84-91.

3) Cinà, C.S., Althani, H, Pasenau, J, and Abouzahr, L. Kommerell's diverticulum and right-sided aortic arch: a cohort study and review of the literature. J Vasc Surg 2004;39:131-139

4) Idrees, J, Keshavamurthy, S, Subramanian, S, Clair, DG, Svensson, LG, and Roselli,EE. Hybrid repair of Kommerell diverticulum. J Thorac Cardiovasc Surg 2014; 147: 973-976

5) Takahashi S, Katayama K, Takasaki T, Sueda T. One stage hybrid procedure for aberrant subclavian artery and thoracic aneurysm. J Vasc Surg Cases 2015;31:232-235.

6) Ren C, Sun L, Huang L, Lai Y, Yang S, Xu S. Hybrid Procedure for Acute Stanford Type a Aortic Dissection With Aberrant Right Subclavian Artery. J Card Surg. 2015;30(3):274-5.

7) Horikawa K, Nishi H, Sekiya N, Yamada M, Takahashi T. Single-stage total arch replacement with open stent graft for Kommerell's diverticulum with right-sided aortic arch.Innovations (Phila) 2018;13:305.

\section{Figure Legends}

Figure 1: Preoperative three-dimensional computed tomography (A, front view; B, right lateral view) showing the Kommerell diverticulum with an aberrant right subclavian artery (black arrow head) and two saccular thoracic aneurysms (black arrows).

Figure 2: Three-dimensional computed tomography post-total arch replacement with a frozen elephant trunk presenting with good patency of the bypass graft and a complete exclusion of the saccular aortic arch aneurysm.

Figure 3: Post thoracic endovascular aortic repair computed tomography revealed the exclusion of the saccular descending aortic aneurysm. 


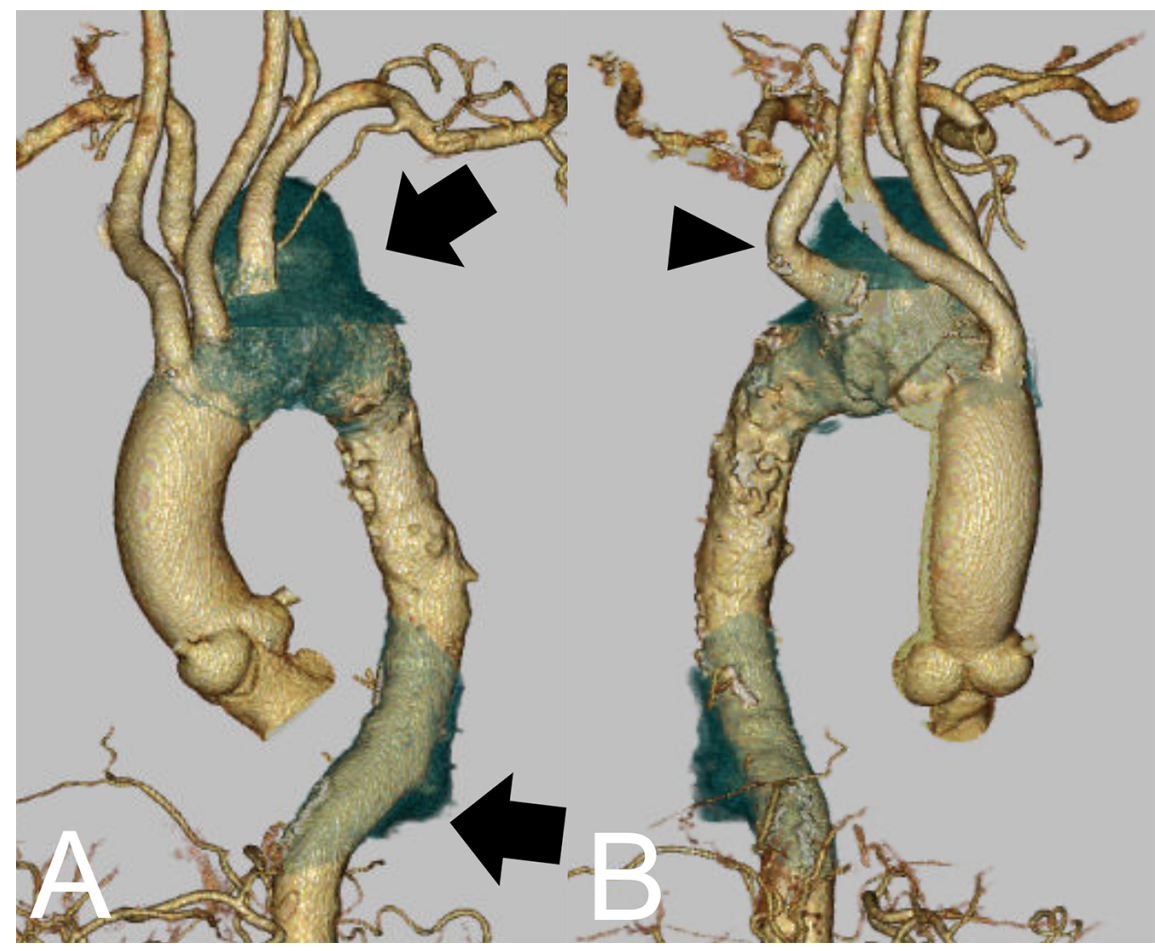




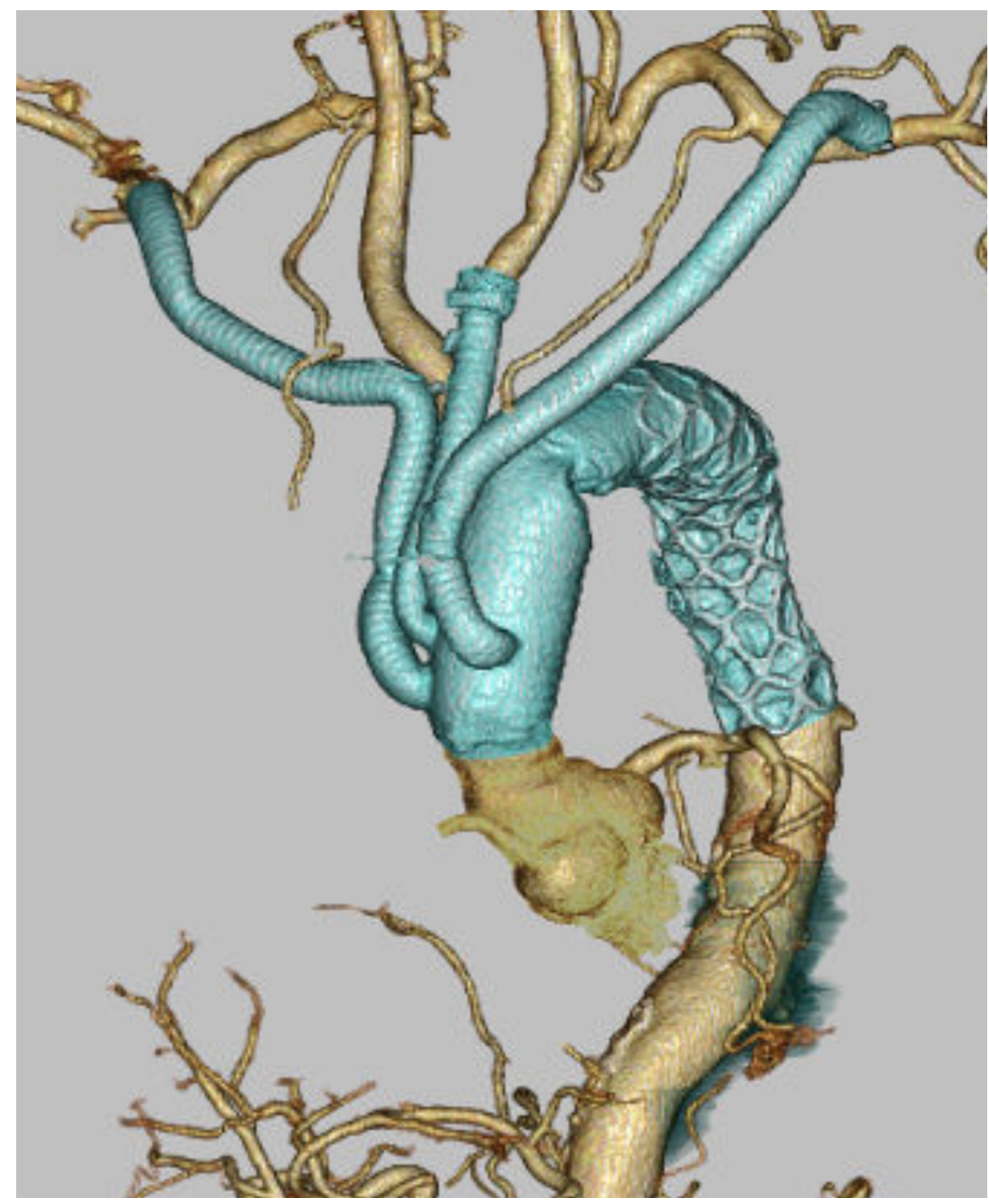




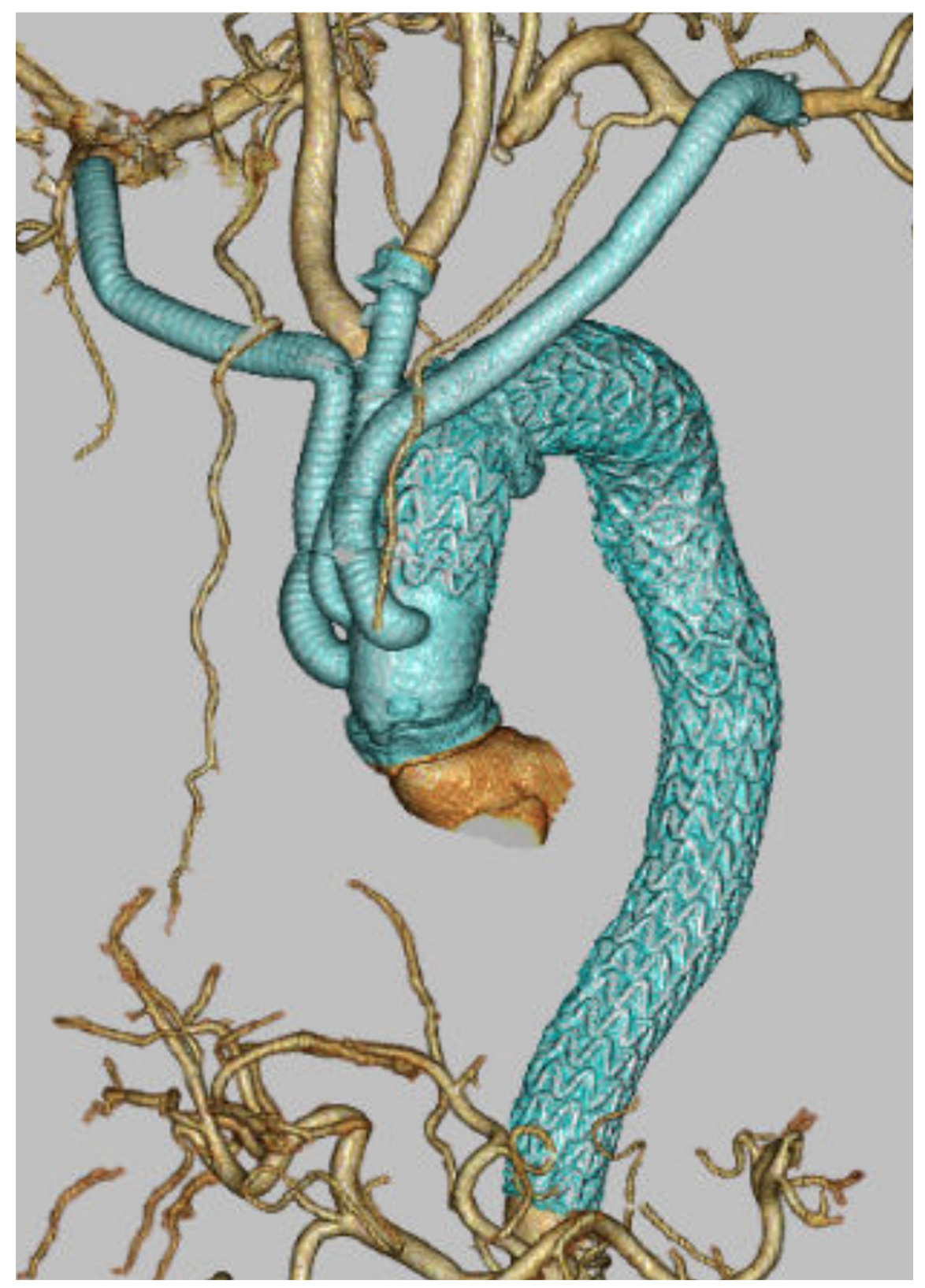

